\title{
Erratum to: The algebraic structure of non-commutative analytic Toeplitz algebras
}

\author{
Kenneth R. Davidson • David R. Pitts
}

Published online: 25 February 2015

(C) Springer-Verlag Berlin Heidelberg 2015

\section{Erratum to: Math. Ann. 311, 275-303 (1998) DOI 10.1007/s002080050188}

The algebraic structure of the non-commutative analytic Toeplitz algebra $\mathfrak{L}_{n}$ is developed in the original article. Some of the results fail for the case $n=\infty$, and this implies that certain other results are not established in this case. In Theorem 3.2 of the original article, we showed there is continuous surjection $\pi_{n, k}$ from $\operatorname{Rep}_{k}\left(\mathfrak{L}_{n}\right)$, the space of completely contractive representations of $\mathfrak{L}_{n}$ into the $k \times k$ matrices $\mathfrak{M}_{k}$, onto the closed unit ball $\overline{\mathbb{B}_{n, k}}$ of $\mathcal{R}_{n}\left(\mathfrak{M}_{k}\right)$ by evaluation at the generators. It is further claimed that if $T=\left[T_{1}, \ldots, T_{n}\right] \in \mathcal{R}_{n}\left(\mathfrak{M}_{k}\right)$ with $\|T\|<1$, then there is a unique representation in $\pi_{n, k}^{-1}(T)$. Further information is obtained for $k=1$ in Theorem 3.3 of the original article. Our proof of these results is valid for $n<\infty$, however, for $n=\infty$ the uniqueness claim is incorrect. An example due to Michael Hartz (see [2, Example 2.4]) shows that $\pi_{\infty, 1}^{-1}(0)$ is very large-it contains a copy of the $\beta \mathbb{N} \backslash \mathbb{N}$.

The difficulty in the proof of Theorems 3.2 and 3.3 of the original article stems from the use of the factorization $A=W X$ used in Lemma 3.1 of the original article. In the case $n=\infty$, this factorization comes from Corollary 2.9. The problem is that the infinite sum in Corollary 2.9 converges in the strong topology, not the norm topology, so that when the representation $\Phi$ is not strongly continuous (or equivalently,

The online version of the original article can be found under doi:10.1007/s002080050188.

K. R. Davidson

Pure Math. Dept., U. Waterloo, Waterloo, ON N2L-3G1, Canada

e-mail: krdavidson@math.uwaterloo.ca

D. R. Pitts $(\bowtie)$

Math. \& Stat. Dept., University of Nebraska, Lincoln, NE 68588, USA

e-mail: dpitts@math.unl.edu 
WOT-continuous), the calculation of the norm in the last lines of the proof of Lemma 3.1 is invalid.

Theorem 3.3 is used throughout Section 4 of the original article. The results of Section 4 are valid when $n<\infty$. However, we are no longer certain of the validity of the following results when $n=\infty$ : Theorem 4.1, Proposition 4.3, Theorem 4.6, Theorem 4.7, and Corollary 4.12. Proposition 4.3 is used in Theorem 4.6 to establish that all automorphisms are WOT-continuous. When attention is restricted to the class of WOT-continuous automorphisms, our proofs of Theorem 4.1 and Theorem 4.7 remain valid when $n=\infty$. We do not know whether WOT-continuity is automatic.

It is worth noting that the results of Section 4 are valid for the class of isometric isomorphisms even when $n=\infty$. This is because any isometric automorphism is WOT-continuous. Indeed, by [3, Theorem 3.3], $\mathfrak{L}_{n}$ has a unique predual, $\mathfrak{L}_{n *}$, which sits naturally inside the dual space $\mathfrak{L}_{n}^{*}$. The uniqueness of the predual implies that $\Theta^{*}$ fixes the image of $\mathfrak{L}_{n *}$, so there is a surjective isometry $\Theta_{*}: \mathfrak{L}_{n *} \rightarrow \mathfrak{L}_{n *}$ such that $\Theta=\left(\Theta_{*}\right)^{*}$. Hence $\Theta$ is weak-* continuous. By [1, Corollary 2.12], the weak-* and WOT topologies on $\mathfrak{L}_{n}$ coincide, so $\Theta$ is WOT-continuous.

\section{References}

1. Davidson, K.R., Pitts, D.R.: Invariant subspaces and hyper-reflexivity for free semigroup algebras. Proc. Lond. Math. Soc. 78(2), 401-430 (1999)

2. Davidson, K.R., Hartz, M., Shalit, O.M.: Multipliers of embedded disks. Complex Anal. Oper. Theory (2014). arXiv:1307.3204v3; doi:10.1007/s11785-014-0360-8

3. Davidson, K.R., Wright, A.: Operator algebras with unique preduals. Canad. Math. Bull. 54(3), 411-421 (2011) 